\title{
Epidemiology of physician-diagnosed neuropathic pain in Brazil
}

This article was published in the following Dove Press journal: Journal of Pain Research

\author{
Margarita Udall' \\ Ian Kudel ${ }^{2}$ \\ Joseph C Cappelleri ${ }^{3}$ \\ Alesia Sadosky' \\ Kristen King-Concialdi ${ }^{2}$ \\ Bruce Parsons' \\ Patrick Hlavacek' \\ Markay Hopps' \\ P Arline Salomon ${ }^{4}$ \\ Marco D DiBonaventura ${ }^{2}$ \\ Patricia Clark ${ }^{5,6}$ \\ João Batista Santos Garcia ${ }^{7}$ \\ 'Pfizer Inc, New York, NY, USA; \\ ${ }^{2}$ Health Outcomes Practice, Kantar \\ Health, New York, NY, USA; ${ }^{3}$ Pfizer \\ Inc, Groton, CT, USA; ${ }^{4}$ Pfizer Inc, \\ Bosques de las Lomas, Mexico; \\ ${ }^{5}$ Clinical Epidemiology Unit, Hospital \\ Infantil de México Federico Gómez, \\ Mexico City, Mexico; ${ }^{6}$ Faculty of \\ Medicine UNAM, Mexico City, Mexico; \\ ${ }^{7}$ Pain and Palliative Care Department, \\ Federal University of Maranhão, \\ Maranhão, Brazil
}

Correspondence: Kristen King-Concialdi Kantar Health, II Madison Ave \# 12, New York, NY 10010, USA

Tel +l 2127063972

Email kristen.concialdi@kantarhealth.com
Objectives: Estimate the prevalence of neuropathic pain $(\mathrm{NeP})$ among chronic pain patients attending Brazilian hospitals and pain clinics in São Paulo, Ceara, and Bahia and explore clinical characteristics by subtypes: painful diabetic peripheral neuropathy ( $\mathrm{pDPN}$ ), central neuropathic pain (CNP), chronic low back pain with a neuropathic component (CLBP-NeP), postherpetic neuralgia (PHN), post-traumatic neuropathic pain (PTN), and post-surgical neuropathic pain (PSN).

Methods: Physicians screened patients reporting chronic pain for $\geq 3$ months $(n=2,118)$ for probable NeP, using the Douleur Neuropathique 4 questionnaire and physician assessment, and reported their NeP subtype(s), symptoms, and medications. Identified NeP patients completed a questionnaire including treatment experiences, quality of life EuroQol 5 Dimensions [EQ-5D]), pain severity and interference (Brief Pain Inventory [BPI]), and Work Productivity and Activity Impairment scales. Descriptive analyses were performed by NeP subtype.

Results: The prevalence of probable NeP was $14.5 \%(\mathrm{n}=307)$. NeP patients were mostly female $(80.5 \%)$, middle-aged (mean $[\mathrm{M}]=52.5, \mathrm{SD}=13.9$ ), and Pardo (44.3\%). Of those diagnosed with an NeP subtype ( $\mathrm{n}=209)$, the largest proportions were CLBP-NeP $(36.8 \%)$, followed by pDPN (18.7\%), CNP (17.7\%), PTN (17.2\%), PSN (13.4\%), and PHN (3.3\%). Across subtypes, the most widely reported symptoms were numbness (range: $62.2 \%-89.7 \%$ ) and hyperalgesia (range: $32.1 \%-76.9 \%$ ) and the most commonly prescribed pain analgesics were NSAID (range: 18.2\%-57.1\%), opioids (range: 0.0\%-39.3\%), and antiepileptics (range: $18.2 \%-57.1 \%$ ). PTN and PSN patients reported the least favorable EQ-5D index scores $(\mathrm{M}=0.42, \mathrm{SD}=0.19)$ and BPI-Pain Severity scores ( $M=7.0, S D=1.9)$, respectively. Those diagnosed with CNP had the least favorable BPI-Pain Interference scores $(M=6.0, S D=2.7)$. Patients with PHN reported the least impairment based on EQ-5D index scores $(\mathrm{M}=0.60, \mathrm{SD}=0.04)$. Those with $\mathrm{pDPN}$ had the most favorable BPI scores (BPI-Pain Severity: $\mathrm{M}=4.6, \mathrm{SD}=2.3$; BPI-Pain Interference: $\mathrm{M}=4.7$, $\mathrm{SD}=2.7$ ).

Conclusion: Evaluation of chronic pain patients in Brazil yielded a $14.5 \%$ probable $\mathrm{NeP}$ prevalence. NSAIDs and opioids were commonly used, and there was a high incidence of NePrelated symptoms with varying levels of dysfunction across subtypes.

Keywords: neuropathic pain, Brazil, quality of life, pain, work productivity and activity impairment

\section{Introduction}

Neuropathic pain $(\mathrm{NeP})$ is defined as "pain arising as a direct consequence of a lesion or disease of the somatosensory system"1 and can be the result of a variety of conditions, including metabolic disease, infection, malignancy, trauma, medications, and toxins. ${ }^{2}$ 
It is a highly prevalent condition that affects a significant proportion of the population. A 2014 systematic review by van Hecke et $\mathrm{al}^{3}$ reported a prevalence rate of $6.9 \%-10.0 \%$ across studies globally, primarily the USA and Europe, with similar rates reported in a separate US-based study by DiBonaventura et al. ${ }^{4}$ It is possible that these rates underestimate the true prevalence of NeP, as no standardized and accepted diagnostic criteria exist for this condition, leading to possible underdiagnosis and undertreatment. ${ }^{5}$

Patients with NeP experience a variety of sensory symptoms, including allodynia, hyperalgesia, paraesthesia, and sensations of burning or freezing. ${ }^{6}$ The impairment associated with these symptoms has been reported across a variety of quality of life and economic domains. ${ }^{7,8}$ Andrew et al reported that, across studies, there was a significant positive relationship between $\mathrm{NeP}$ severity and both work-related impairment and healthcare resource utilization (HCRU). ${ }^{9}$ A recent European study of nearly 4,000 patients reported increased HCRU and high direct costs, as well as lost work productivity (indirect costs). ${ }^{10}$ The authors found that total costs per patient ranged from 9,305 euros in Italy to 14,446 euros in Germany, annually, with the majority dedicated to indirect costs associated with care.

Despite this, few studies have examined the prevalence of NeP in Brazil or examined NeP-related outcomes. Those studies that do exist have reported NeP estimates of $60 \%$ among those with chronic pain ${ }^{11}$ and $10 \%$ among the general population. ${ }^{12}$ Additionally, there does not appear to be any studies reporting the impairment associated with specific NeP etiologies. The current study estimates the prevalence of probable NeP among chronic pain patients attending Brazilian hospitals and pain clinics in São Paulo, Ceara, and Bahia and assesses the clinical characteristics of the six principal NeP subtypes: painful diabetic peripheral neuropathy (pDPN), central neuropathic pain (CNP), chronic low back pain with a neuropathic component (CLBP-NeP), postherpetic neuralgia $(\mathrm{PHN})$, post-traumatic neuropathic pain $(\mathrm{PTN})$, and post-surgical neuropathic pain (PSN).

\section{Methods}

\section{Participants}

Patients who presented with pain (as a primary or secondary complaint) and answered affirmatively to experiencing self-reported chronic pain ( $\geq 3$ months within the past 12 months) at either of two urban general hospitals (Faculdade de Medicina do ABC [Santo André, São Paulo] and Instituto de Estudos e Pesquisas Clínicas do Ceará-IEPCE [Fortaleza, Ceará]) or one urban pain clinic (Clínica de Terapia de Dor LTDA [Salvador, Bahia]) were invited to participate in the study by physicians/investigators. Physicians $(n=16)$ were recruited by researchers from the same hospitals and pain clinic and were either licensed primary care provider generalists $(n=8)$ or specialized in a predefined domain, including orthopedics $(n=1)$ and endocrinology $(n=2)$. There were also five pain specialists, of which three were orthopedic specialists, an anesthesiologist, and a neurologist. All clinicians spent at least $75 \%$ of their time treating patients and felt confident diagnosing NeP. The study was approved by each participating site's regulatory board and the National Ethics Committee (CONEP: Opinion Number 1.440.502), which regulates all research involving human participants in Brazil. All patients provided written informed consent; however, as per local laws, participants were not provided compensation for participating in the study.

\section{Physician examination}

Physicians evaluated each patient who met the criteria for self-reported chronic pain. To replicate real-world conditions, physicians followed their own procedures for collecting patients' history and conducting a physical examination to ascertain whether a diagnosis of NeP was warranted. However, by definition, central NeP involves conditions or injuries associated with central nervous system lesions (ie, central poststroke pain, multiple sclerosis, and spinal cord injury-related NeP). Those with central NeP included patients with nondiabetic NeP, although some patients with nondiabetic NeP could have instead been diagnosed with a different specific NeP subtype (except for pDPN), as determined by the physician. For patients in whom a NeP diagnosis was suspected and those who agreed to the informed consent, physicians asked patients the Douleur Neuropathique 4 (DN4) questions. ${ }^{13}$ For the DN4, a score of $\geq 4$ indicates that the pain is likely to be NeP. This cutoff was used with the physician's assessment to determine if a patient was diagnosed with probable NeP or not. For those diagnosed with probable NeP, physicians also reported the presence of symptoms (eg, areas of numbness, allodynia), whether patients were diagnosed with a subtype (pDPN, CNP, CLBP-NeP, PHN, PTN, PSN), comorbid medical conditions, and prescription medications that the patient had been prescribed for their NeP within the last 6 months. These patients also completed an additional 20-minute survey that included questions regarding sociodemographic characteristics and instruments assessing pain severity and interference with 
function, health status, and impairment in work productivity and daily activities.

\section{Measures}

\section{Sociodemographic characteristics and health behaviors}

Sex, age, ethnic background, household income, education, employment status, insurance status, body mass index, and self-reported nonprescription therapy for pain management were assessed.

\section{Health status}

The EuroQol 5 Dimensions (EQ-5D) was used as the measure of quality of life. ${ }^{14}$ The EQ-5D is a five-item instrument that asks respondents to rate their health status that day. One component includes a VAS that ranges from 0 to 100 (higher scores are better) and gauges the respondents' perception of their overall health on the day the survey was completed. The five items were converted into a health utility index using the Brazilian value set (range: -0.11 to 1.0 , where 0 is death and 1 is perfect health). ${ }^{15}$

\section{Pain severity and interference}

The Brief Pain Inventory (BPI) was used to assess pain severity (BPI-Pain Severity, four items) and pain interference (BPIPain Interference, seven items). ${ }^{16}$ The BPI asks respondents to rate the intensity of the pain they are currently experiencing, as well as the intensity of pain experienced within the prior 24 hours, at the worst, at the least, and on average, using response options ranging from 0 (no pain) to 10 (pain as bad as you can imagine). The BPI also asks respondents to rate on a scale from 0 (does not interfere) to 10 (interferes completely), the degree to which their pain interferes with functioning in seven domains: general activity, walking, mood, sleep, work, relations with other persons, and enjoyment of life. Subscale scores are derived by taking the mean of the scores within each subscale, with higher scores indicative of greater pain severity and interference. ${ }^{16}$

\section{Work and activity impairment}

The six-item Work Productivity and Activity Impairment Questionnaire: Specific Health Problem v2.0 (WPAI-SHP) questionnaire was used to measure the effect of pain on work and leisure activities among those participants who were employed. The WPAI-SHP possesses four subscales that each yields a percentage-based level of impairment (0\%-100\%). Three are work related: absenteeism, presenteeism, and overall work impairment. The activity impairment scale measures non-work-related activity. ${ }^{17}$

\section{Douleur Neuropathique 4}

Physicians administered the DN4 to patients presenting with chronic pain to estimate the probability of NeP. The DN4 is a 10-item instrument that measures the presence of different characteristics of pain (eg, burning, painful cold). Having at least four of these characteristics suggests a diagnosis of $\mathrm{NeP}$; a cutoff of 4 (range: 0 to 10 ) has demonstrated $80 \%$ and $92 \%$ sensitivity and specificity, respectively. ${ }^{13}$ The DN4 has been linguistically validated for Brazilian Portuguese. ${ }^{18}$

\section{Analysis}

The overall prevalence of probable NeP (based on the DN4 score), along with 95\% CI, was reported among all respondents who presented with chronic pain. Sociodemographic and comorbid conditions were reported for all participants diagnosed with NeP. NeP symptoms, medication use, and patient-reported outcomes (health status, pain severity and interference, and work productivity) were reported for each diagnosed subgroup of NeP. Categorical data were reported using numbers and percentages. Continuous data were reported using mean $(\mathrm{M})$ and $\mathrm{SD}$.

\section{Results Descriptive data}

The current study enrolled 2,118 participants who reported pain, as a primary or secondary complaint, that lasted for $\geq 3$ months of the previous 12 months. Of those, 307 were diagnosed with probable NeP by a physician and had a DN4 score of $\geq 4$. The overall prevalence of $\mathrm{NeP}$ was estimated to be $14.5 \%$ (95\% CI: $13.0 \%-16.0 \%)$. The majority of those enrolled in the study were female $(n=1,597,75.4 \%)$, middleaged (50.1 years, $S D=16.3)$, and Pardo $(n=648,30.6 \%$; Table 1). Among those diagnosed with probable $\mathrm{NeP}$, the majority were also female $(n=247,80.5 \%)$ and Pardo $(136,44.3 \%)$, with a slightly higher mean age of $52.5(\mathrm{SD}=13.9)$ years.

Of those 307 individuals identified by their physician and DN4 score as having probable NeP, 209 were diagnosed by a physician with a specific $\mathrm{NeP}$ subtype, with the largest proportion diagnosed with CLBP-NeP $(n=77,36.8 \%)$. Others were diagnosed with pDPN ( $\mathrm{n}=39,18.7 \%)$, CNP $(\mathrm{n}=37$, $17.7 \%)$, PTN ( $n=36,17.2 \%)$, followed by PSN ( $n=28,13.4 \%)$ and $\mathrm{PHN}(\mathrm{n}=7,3.3 \%)$. Of those diagnosed with a specific NeP subtype, 194 (92.8\%) were diagnosed with one subtype and 15 were diagnosed with two $(7.2 \%)$. 
Table I Demographic characteristics for the total and NeP sample

\begin{tabular}{|c|c|c|c|}
\hline Characteristics & All patients & $\begin{array}{l}\text { NeP } \\
\text { participants }\end{array}$ & $\begin{array}{l}\text { NeP participants } \\
\text { with subtype known }\end{array}$ \\
\hline \multicolumn{4}{|l|}{ Categorical variables } \\
\hline $\mathrm{N}$ & 2,118 & 307 & 209 \\
\hline \multicolumn{4}{|l|}{ Sex } \\
\hline Female & I,597 (75.4\%) & 247 (80.5\%) & $162(77.6 \%)$ \\
\hline \multicolumn{4}{|l|}{ Ethnic background } \\
\hline White (Branco) & $437(20.6 \%)$ & $83(27.0 \%)$ & $55(26.3 \%)$ \\
\hline Black (Preto) & $106(5.0 \%)$ & $63(20.7 \%)$ & $47(22.5 \%)$ \\
\hline Yellow (Amarelo) & $4(0.2 \%)$ & $2(0.1 \%)$ & $0(0.0 \%)$ \\
\hline Brown (Pardo) & $648(30.6 \%)$ & $136(44.3 \%)$ & $92(44.0 \%)$ \\
\hline Native (Indigena) & $2(0.1 \%)$ & $2(0.1 \%)$ & $2(1.0 \%)$ \\
\hline \multicolumn{4}{|l|}{ Employment } \\
\hline Full-time, part-time, self-employed & & $96(31.9 \%)$ & $58(28.2 \%)$ \\
\hline \multicolumn{4}{|l|}{ Household income } \\
\hline$>\$ 3,067.41$ & & $16(5.2 \%)$ & II (5.3\%) \\
\hline$\$ 2,352.90$ to $\$ 3,067.4 \mathrm{I}$ & & $15(4.9 \%)$ & $8(3.8 \%)$ \\
\hline$\$ 545.82$ to $\$ 2,352.89$ & & 69 (22.5\%) & $49(23.4 \%)$ \\
\hline$\$ 341.53$ to $\$ 545.81$ & & $5 \mathrm{I}(16.6 \%)$ & $31(14.8 \%)$ \\
\hline$\leq \$ 341.52$ & & $133(43.3 \%)$ & $93(44.5 \%)$ \\
\hline \multicolumn{4}{|l|}{ Education } \\
\hline Primary incomplete & & $45(14.7 \%)$ & $28(13.4 \%)$ \\
\hline Primary school complete & & $12(3.9 \%)$ & 9 (4.3\%) \\
\hline Elementary incomplete & & 27 (8.8\%) & $21(10.0 \%)$ \\
\hline Elementary complete & & $14(4.6 \%)$ & II (5.3\%) \\
\hline High school incomplete & & $17(5.5 \%)$ & $13(6.2 \%)$ \\
\hline High school complete & & $89(29.0 \%)$ & $63(30.1 \%)$ \\
\hline Degree incomplete & & $21(6.8 \%)$ & $16(7.7 \%)$ \\
\hline Degree complete & & $53(17.3 \%)$ & 31 (14.8\%) \\
\hline Master/doctorate & & 9 (2.9\%) & $3(1.4 \%)$ \\
\hline \multicolumn{4}{|l|}{ Marital status } \\
\hline Married & & | 48 (48.2\%) & II 4 (54.5\%) \\
\hline Single, never married & & $62(20.2 \%)$ & $39(18.7 \%)$ \\
\hline Divorced & & $20(6.5 \%)$ & $6(2.9 \%)$ \\
\hline Separated & & $14(4.6 \%)$ & $8(3.8 \%)$ \\
\hline Widowed & & $25(8.1 \%)$ & $17(8.1 \%)$ \\
\hline Living with partner & & $16(5.2 \%)$ & $10(4.8 \%)$ \\
\hline \multicolumn{4}{|l|}{ Continuous variables } \\
\hline Age, mean (SD) & $50.1(16.3)$ & $52.5(13.9)$ & $53.0(14.3)$ \\
\hline
\end{tabular}

Notes: Due to missing data, numbers may not sum to $100 \%$. Data for sex, ethnic background, and age were collected on all participants, whereas data on employment, household income, education, and marital status were only collected from patients with $\mathrm{NeP}$.

Abbreviation: NeP, neuropathic pain.

Across NeP subtypes, high blood pressure was consistently one of the most reported comorbid conditions (Figure 1) and the most common for patients with CNP ( $n=13$; $35.1 \%)$ and CLBP-NeP $(\mathrm{n}=30 ; 39.0 \%)$. As expected, the clear majority ( $n=38 ; 97.4 \%)$ of those diagnosed with $\mathrm{pDPN}$ reported comorbid type 1 or type 2 diabetes. Insomnia/sleep difficulties ( $\mathrm{n}=8 ; 22.2 \%$ ) were more commonly reported than high blood pressure in those diagnosed with PTN. Additionally, of patients with NeP subtype known, 23 (11.0\%) patients reported no comorbid conditions, $44(21.1 \%)$ patients reported one comorbid condition, 33 (15.8\%) reported two comorbid conditions, 38 (18.2\%) reported three comorbid conditions, and 64 (30.6\%) reported four or more comorbid conditions.

As indicated in Figure 2, almost all NeP-related sensory symptoms assessed were prevalent, regardless of specific $\mathrm{NeP}$ etiology. The most commonly reported symptoms were numbness (range: 62.2\%-89.7\%), hyperalgesia (range: $32.1 \%-76.9 \%$ ), allodynia (range: $18.9 \%-57.1 \%$ ), impaired vibratory sense (range: $20.8 \%-51.4 \%$ ), and changes in 


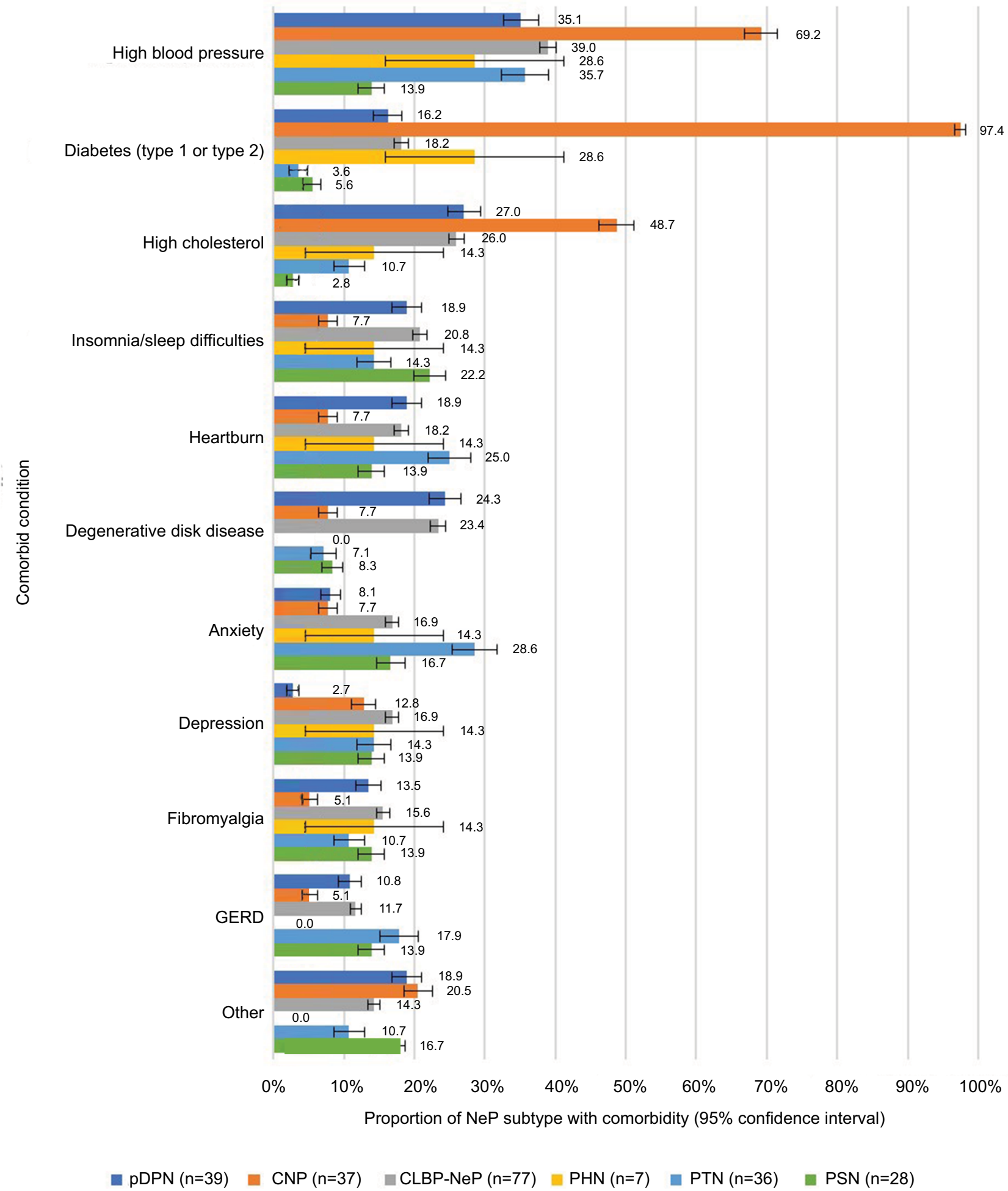

Figure I Comorbid conditions reported by at least 10\% of the patients diagnosed with $\mathrm{NeP}(n=209)$.

Notes: $95 \% \mathrm{Cl}$ values are displayed. Patients could be diagnosed with multiple subtypes; hence, totals add up to $>100 \%$.

Abbreviations: CLBP-NeP, chronic lower back pain with a neuropathic component; CNP, central neuropathic pain; pDPN, painful diabetic peripheral neuropathy; GERD, gastroesophageal reflux disease; NeP, neuropathic pain; PSN, post-surgical neuropathic pain; PHN, postherpetic neuralgia; PTN, post-traumatic neuropathic pain.

thermal perception (range: $7.7 \%-57.1 \%$ ). As noted in Figure 3 , the most frequently prescribed NeP medications in the past 6 months were antiepileptics (range: $18.2 \%-57.1 \%$ ), NSAIDs (range: $14.3 \%-50.0 \%$ ), and opioids (range: $0.0 \%-39.3 \%$ ).
The lowest EQ-VAS scores within each NeP subtype were found for those with CLBP-NeP $(\mathrm{M}=47.0, \mathrm{SD}=22.3)$, with the lowest health utilities among patients with PTN $(\mathrm{M}=0.4, \mathrm{SD}=0.2)$ (Figure 4). Patients with PTN had the 


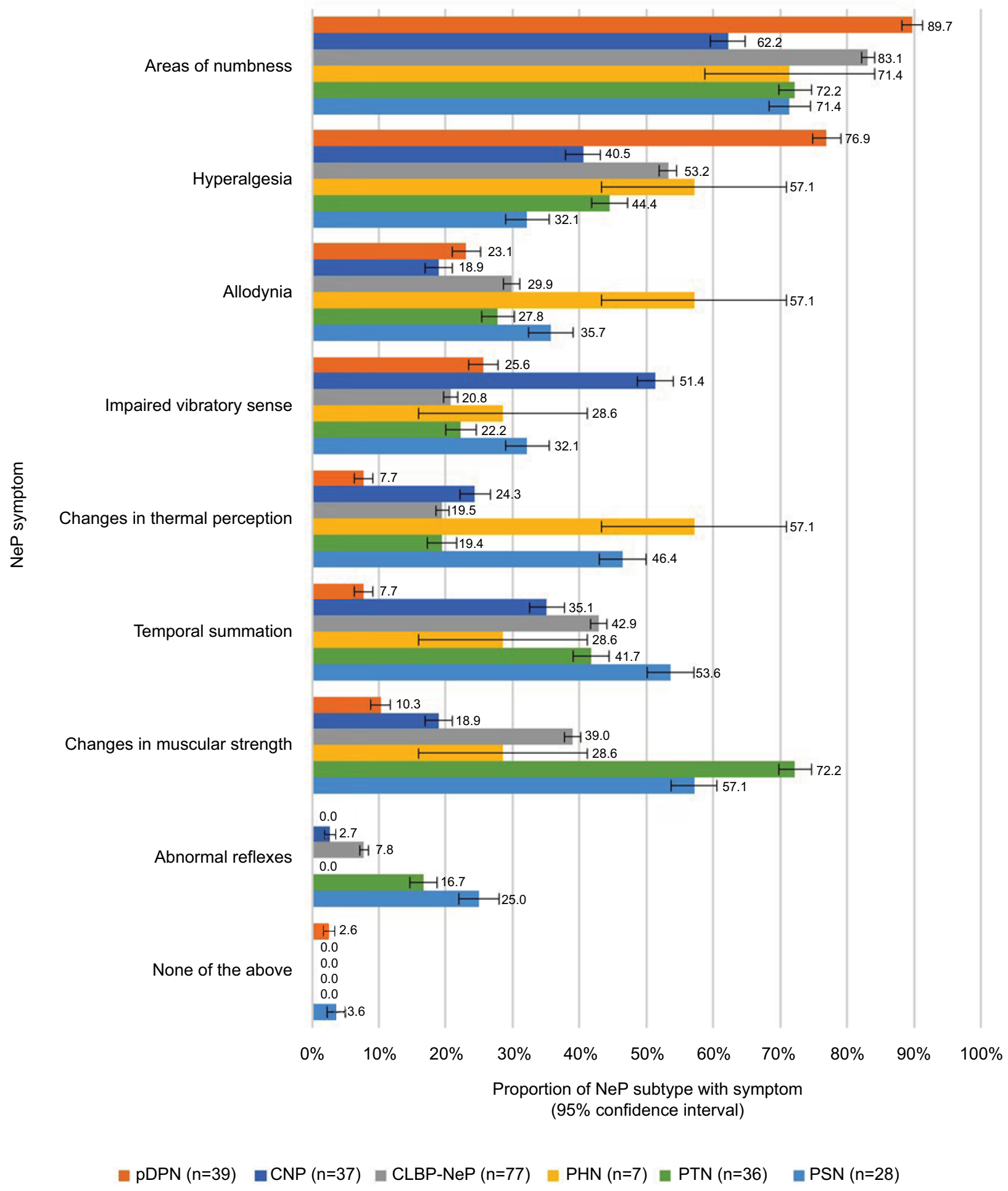

Figure $2 \mathrm{NeP}$ symptoms exhibited by physician-confirmed $\mathrm{NeP}$ patient during the examination.

Notes: $95 \% \mathrm{Cl}$ values are displayed. Totals reflect that patients could be diagnosed with multiple subtypes.

Abbreviations: CLBP-NeP, chronic lower back pain with a neuropathic component; CNP, central neuropathic pain; pDPN, painful diabetic peripheral neuropathy; NeP, neuropathic pain; PSN, post-surgical neuropathic pain; PHN, postherpetic neuralgia; PTN, post-traumatic neuropathic pain.

second lowest EQ-VAS ( $\mathrm{M}=47.4, \mathrm{SD}=23.2)$ with a mean score only slightly higher than those with CLBP-NeP. In contrast, patients with PHN had both the highest EQ-VAS $(\mathrm{M}=67.3, \mathrm{SD}=13.9)$ and health utility scores $(\mathrm{M}=0.60$,
$\mathrm{SD}=0.04)$. Additionally, patients with PHN had the secondlowest BPI-Pain Severity $(\mathrm{M}=5.1, \mathrm{SD}=3.0)$ and BPI-Pain Interference $(\mathrm{M}=5.5, \mathrm{SD}=2.7)$ scores, indicating relatively positive health status and low pain (Figure 5). They also had 


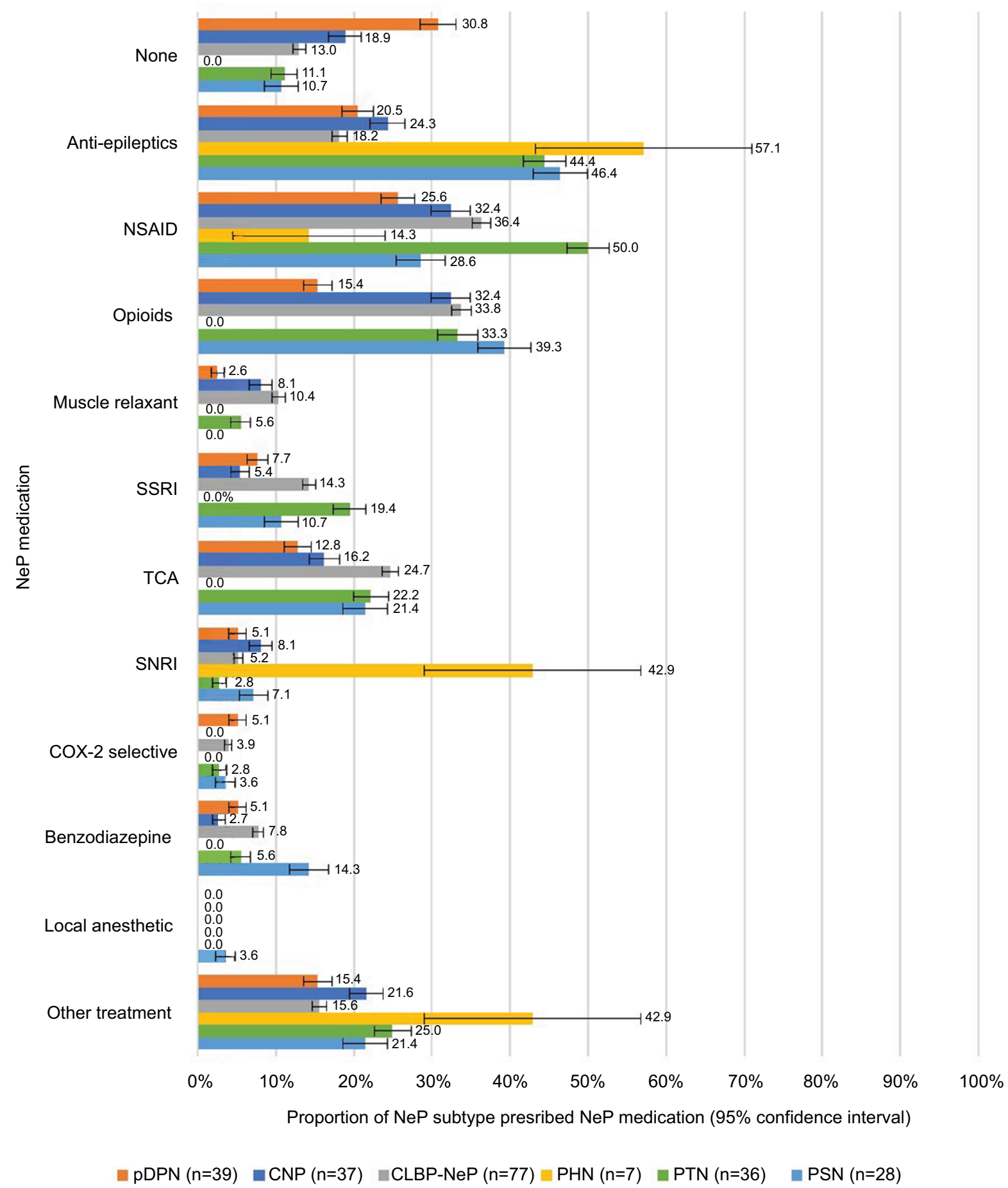

Figure 3 Clinical features of $\mathrm{NeP}$ and the clinical profile of patients for each NeP subtype: medication use. Notes: $95 \% \mathrm{Cl}$ values are displayed. Totals reflect that patients could be diagnosed with multiple subtypes.

Abbreviations: CLBP-NeP, chronic lower back pain with a neuropathic component; CNP, central neuropathic pain; pDPN, painful diabetic peripheral neuropathy; NeP, neuropathic pain; PSN, post-surgical neuropathic pain; PHN, postherpetic neuralgia; PTN, post-traumatic neuropathic pain; SNRI, serotonin-norepinephrine reuptake inhibitors; SSRI, serotonin-specific reuptake inhibitor; TCA, tricyclic antidepressant.

the lowest absenteeism and the second-lowest presenteeism, overall work impairment, and activity impairment (Figure 6). For the BPI, patients with PSN had the highest pain severity scores $(\mathrm{M}=6.6, \mathrm{SD}=1.7)$, and $\mathrm{CNP}$ patients scored the highest pain interference scores ( $\mathrm{M}=6.0, \mathrm{SD}=2.7)$. Conversely, those with pDPN reported the lowest pain severity ( $M=4.6$, $\mathrm{SD}=2.3)$ and pain interference $(\mathrm{M}=4.6, \mathrm{SD}=2.6)$ scores on the BPI. Patients with PTN had the second highest pain severity $(\mathrm{M}=6.4, \mathrm{SD}=1.9)$ and pain interference $(\mathrm{M}=6.0$, $\mathrm{SD}=2.6)$ scores on the BPI. 


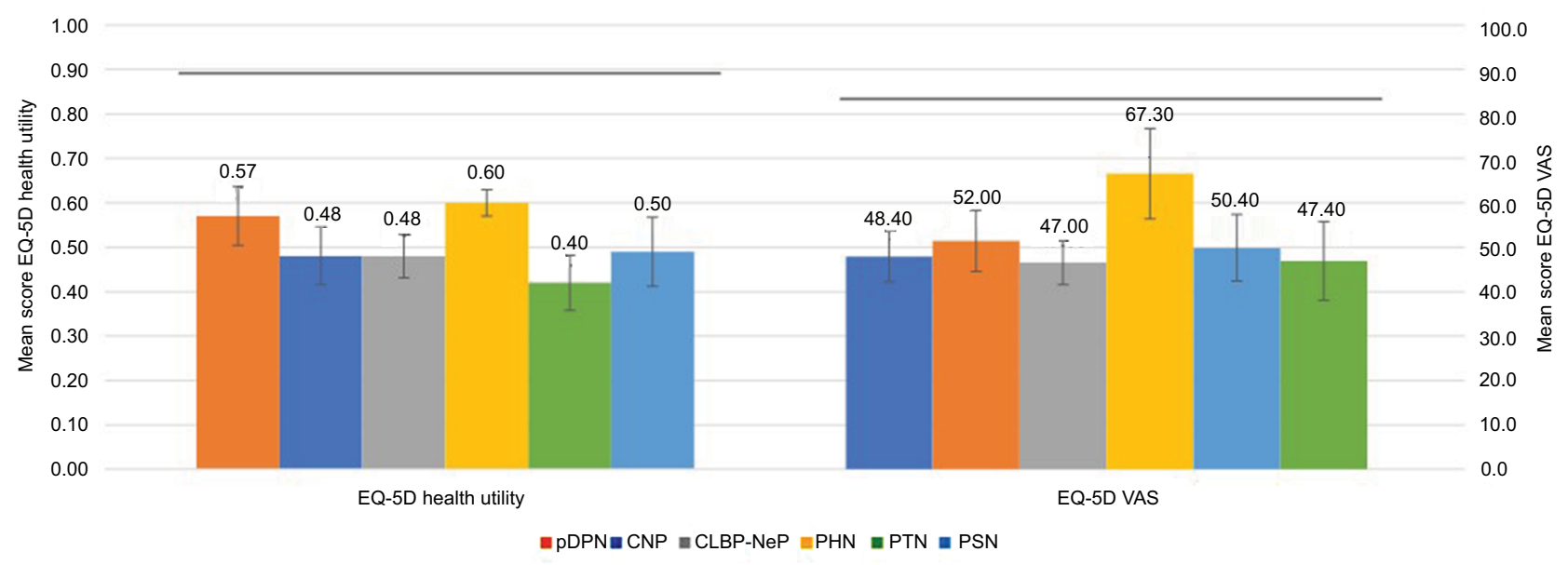

Figure 4 Mean $(95 \% \mathrm{Cl})$ health status and economic burden of patients for each NeP subtype: EQ-5D.

Notes: Mean and $95 \% \mathrm{Cl}$ values are displayed. The Brazil population norms for the EQ-5D scales are overlaid on the figure (Health Utility 0.89; VAS 83.8). ${ }^{28}$

Abbreviations: CLBP-NeP, chronic lower back pain with a neuropathic component; CNP, central neuropathic pain; pDPN, painful diabetic peripheral neuropathy; NeP, neuropathic pain; PSN, post-surgical neuropathic pain; PHN, postherpetic neuralgia; PTN, post-traumatic neuropathic pain.

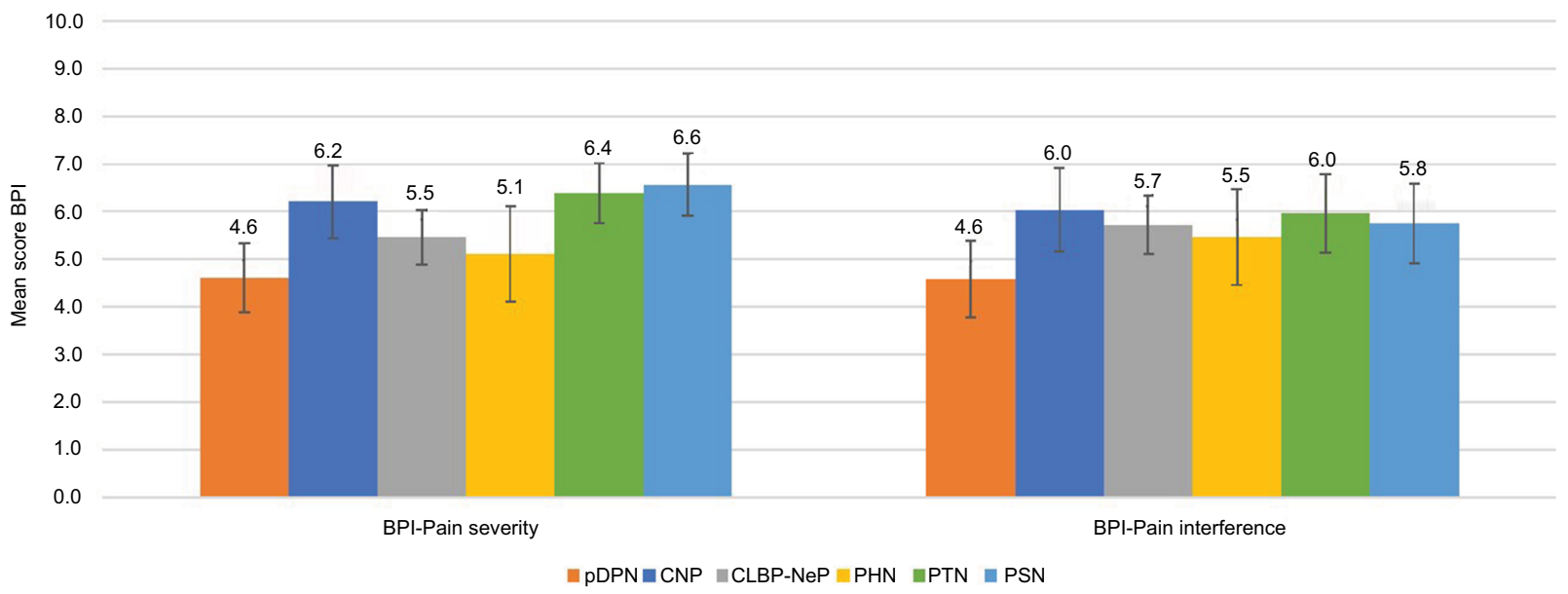

Figure 5 Mean $(95 \% \mathrm{Cl})$ health status and economic burden of patients for each NeP subtype: BPI.

Notes: Mean and $95 \% \mathrm{Cl}$ values are displayed.

Abbreviations: BPI, Brief Pain Inventory; CLBP-NeP, chronic lower back pain with a neuropathic component; CNP, central neuropathic pain; pDPN, painful diabetic peripheral neuropathy; NeP, neuropathic pain; PSN, post-surgical neuropathic pain; PHN, postherpetic neuralgia; PTN, post-traumatic neuropathic pain.

Patients also reported their current employment status. Patients with PHN reported the highest levels of being employed full-time, part-time, or self-employed $(\mathrm{n}=3$, $42.9 \%$ ). The other NeP subtypes reported similar levels of employment status (pDPN: $n=11,28.9 \%$; CNP: $n=10,27.8 \%$; CLBP: $n=21,27.3 \%$; PTN: $n=n=9,25.0 \%$; PSN: $n=7,25.9 \%$ ) (data not shown). Among those employed, patients with CNP experienced the greatest overall work impairment $(\mathrm{M}=77.1 \%$, $\mathrm{SD}=18.2 \%)$ and presenteeism $(\mathrm{M}=74.6 \%, \mathrm{SD}=17.5 \%)$. Absenteeism was highest among those with PTN (M=26.8\%, $\mathrm{SD}=34.9 \%$ ). PSN patients reported the greatest degree of activity impairment ( $M=79.6 \%, \mathrm{SD}=18.9 \%$; see Figure 6).
Across health status, pain, and economic and activity burden, patients with pDPN and/or PHN, on average, did better than those with other NeP subtypes. In contrast, patients with PTN, on average, scored less favorably.

\section{Discussion}

The current study established a probable NeP prevalence rate of $14.5 \%$ among chronic pain patients seeking treatment in a clinical setting. As one might expect, given the current study's clinical context, where physicians are likely to see more severe cases, this is a higher rate than studies that have been conducted in the general population, ${ }^{12,19}$ but 


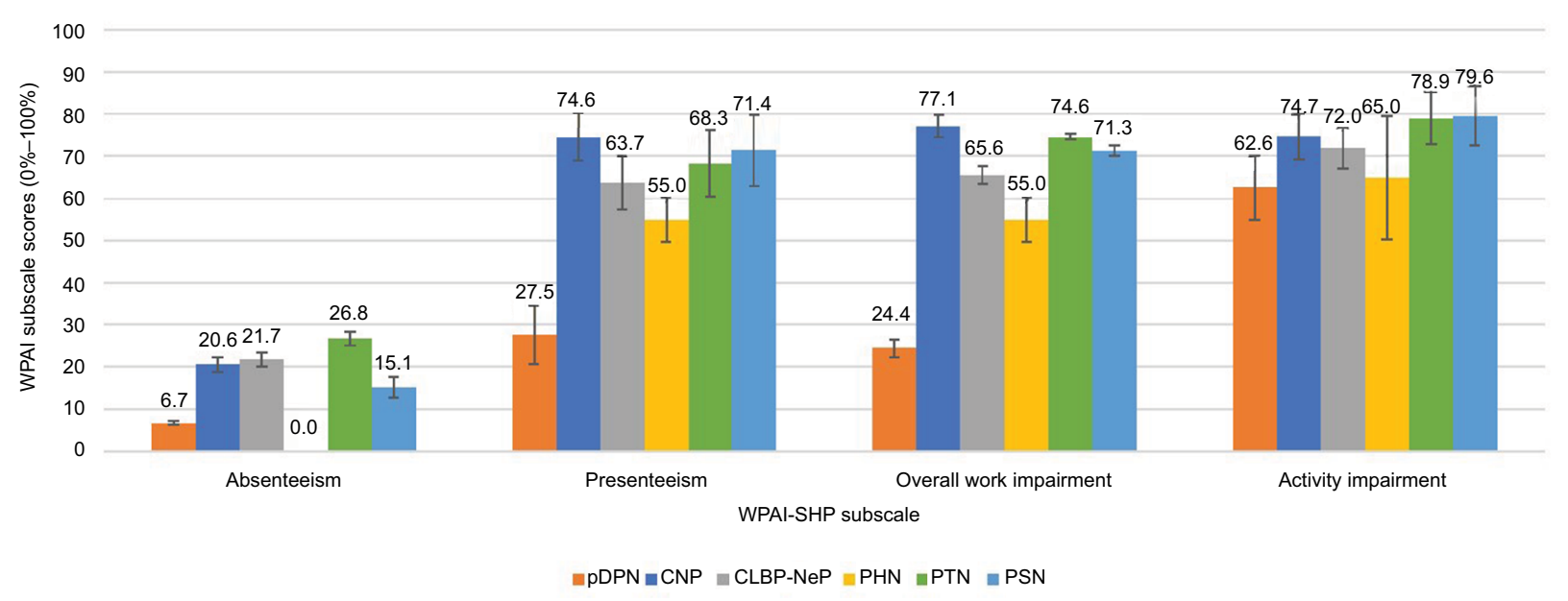

Figure 6 Mean $(95 \% \mathrm{Cl})$ economic and activity burden for each NeP subtype: WPAI-SHP.

Notes: Mean and $95 \% \mathrm{Cl}$ values are displayed.

Abbreviations: CLBP-NeP, chronic lower back pain with a neuropathic component; CNP, central neuropathic pain; pDPN, painful diabetic peripheral neuropathy; NeP, neuropathic pain; PSN, post-surgical neuropathic pain; PHN, postherpetic neuralgia; PTN, post-traumatic neuropathic pain; WPAI-SHP, Work Productivity and Activity Impairment Questionnaire: Specific Health Problem v2.0.

it is also slightly higher than the $10 \%$ prevalence found in a 2009-2010 sample of Sao Luis, Brazil. ${ }^{19}$ A comparison of sample sizes shows that $\mathrm{NeP}$ was most frequently identified in middle-aged and Pardo patients. Consistent with a previous US-based study, ${ }^{4}$ the most frequently diagnosed condition in this study was CLBP-NeP (25.1\%), followed by similar rates of pDPN (12.7\%), CNP (12.5\%), and PTN $(11.7 \%)$. As has been noted in previous epidemiological research, ${ }^{20}$ patients reported high rates of comorbid disease and NeP-related symptomatology across all subtypes, most notably high blood pressure and high cholesterol, and areas of numbness and hyperalgesia. Once again, and consistent with previous research, ${ }^{4,21-23}$ the majority of patients across all subtypes reported currently being treated, specifically using prescription NSAIDs, antiepileptics, and opioids to treat their pain. The reliance upon NSAIDs and opioids may stem from patients also managing nociceptive pain, eg, osteoarthritis, which would also be consistent with previous studies. ${ }^{8,21,22,24-26}$ Currently, there is a lack of systematization in the treatment of $\mathrm{NeP}$ with respect to published guidelines.

Patients with a physician-confirmed diagnosis reported consistent impairments in quality of life across all subtypes, with those diagnosed with PTN possessing the lowest health utility score, in contrast to a previous meta-analysis that established CNP patients as the group with the greatest level of impairment. ${ }^{27}$ Pain severity and interference were notable across all subtypes of $\mathrm{NeP}$ and generally within the same range. This is notable as the greater intensity of pain contributes to interference in daily life, making it more difficult to manage than non-NeP. Patients also reported significant and similar levels of activity impairment, with the one exception being those diagnosed with pDPN, who reported substantially lower overall work impairment and presenteeism. A previous study assessing work productivity and activity impairment in persons with any type of $\mathrm{NeP}$ in Brazil found that on average absenteeism was $26.0 \%$, presenteeism was $59.7 \%$, overall work impairment was $59.8 \%$, and activity impairment was $60.4 \% .{ }^{19}$ All but activity impairment fell within the range of the NeP subtype mean values. This is, however, novel in that it assessed work productivity and activity impairment across the various $\mathrm{NeP}$ subtypes.

\section{Limitations}

The current study utilized patient self-reported information for outcomes; thus, responses were subject to recall and self-presentation biases. Furthermore, whereas the utilization of multiple physicians and recruitment sites increased the representativeness of patients sampled (including the corresponding variation in clinical practices), some bias may have been introduced via variations in data collection processes across sites, despite the training provided to all physicians and study staff. Furthermore, although data were collected from multiple sites, all were in urban areas, and results may not generalize to those NeP patients living outside these specific urban areas or to the broader Brazilian population, including those seeking treatment in rural areas. It is likely that certain groups of patients, namely those who do not visit physicians regularly, were underrepresented in this study. It must also be noted that the two largest groups of patients surveyed were those with neuropathic back pain and those with diabetic 
neuropathy. Given the variation in symptomology and patient behavior among these two groups, it is important to recognize the heterogeneity that exists within the study sample. A final limitation is the cross-sectional nature of the study design, which limited the ability to infer any causal conclusions.

\section{Conclusion}

The prevalence of probable $\mathrm{NeP}$ among chronic pain patients receiving treatment in a pain or general clinic or hospital in Brazil is $\sim 14.5 \%$. In general, and consistent with previous research beyond Brazil, all patient subtypes reported impairment and varying levels of dysfunction across a range of measures. The current study provided important insight into the experience of NeP patients in Brazil and reinforced the debilitating nature of this condition.

\section{Acknowledgments}

The authors wish to acknowledge the literature review and editing assistance of Errol J. Philip, PhD, and Shawna R. Calhoun, MPH, on behalf of Kantar Health, with funding from Pfizer, Inc. The study described in this manuscript was funded by Pfizer, Inc.

The abstract of this paper was presented at the meeting of the International Society for Pharmacoeconomics and Outcomes Research - Latin America, São Paulo, Brazil, as a poster presentation with interim findings. The poster's abstract has been published. ${ }^{29}$

\section{Disclosure}

MU, JCC, AS, BP, PH, MH, and PAS are employees and stockholders of Pfizer, Inc., which funded the current study. $\mathrm{KK}-\mathrm{C}$ is an employee of Kantar Health, which received funding from Pfizer, Inc., for conducting and reporting on the current study and for manuscript development. MDB was a Kantar Health employee during the time the study was conducted and is a current employee of Pfizer and was involved in manuscript development as a Pfizer employee. IK was employed by Kantar Health at the time this study was conducted. The other authors report no other conflicts of interest in this work.

\section{References}

1. International Association for the Study of Pain. Neuropathic Pain; 2014. Available from: http://www.iasp-pain.org/Education/Content.aspx?Item Number=1698\&navItemNumber=576-Neuropathicpain. Accessed July 3, 2015.

2. Charlton JE, editor. Core Curriculum for Professional Education in Pain. 3rd Ed. Seattle: IASP Press; 2005.

3. van Hecke O, Austin SK, Khan RA, Smith BH, Torrance N. Neuropathic pain in the general population: a systematic review of epidemiological studies. Pain. 2014;155(4):654-662.
4. Dibonaventura M, Sadosky A, Concialdi K, et al. The prevalence of broad neuropathic pain in the United States: results from a multi-channel general population health survey. J Pain Res. 2016;17(4 Suppl):S11.

5. Haanpää ML, Backonja MM, Bennett MI, et al. Assessment of neuropathic pain in primary care. Am J Med. 2009;122(10 Suppl): S13-S21.

6. Smith BH, Torrance N. Neuropathic pain. In: Croft P, Blyth FM, van der Windt D, editors. Chronic Pain Epidemiology. New York: Oxford University Press;2010:209-234.

7. Smith BH, Torrance N. Epidemiology of neuropathic pain and its impact on quality of life. Curr Pain Headache Rep. 2012;16(3):191-198.

8. Schaefer C, Sadosky A, Mann R, et al. Pain severity and the economic burden of neuropathic pain in the United States: BEAT Neuropathic Pain Observational Study. Clinicoecon Outcomes Res. 2014;6:483-496.

9. Andrew R, Derry S, Taylor RS, Straube S, Phillips CJ. The costs and consequences of adequately managed chronic non-cancer pain and chronic neuropathic pain. Pain Pract. 2014;14(1):79-94.

10. Liedgens H, Obradovic M, de Courcy J, Holbrook T, Jakubanis R. A burden of illness study for neuropathic pain in Europe. Clinicoecon Outcomes Res. 2016;8:113-126.

11. Vieira AS, Baptista AF, Mendes L, et al. Impact of neuropathic pain at the population level. J Clin Med Res. 2014;6(2):111-119.

12. de Moraes Vieira EB, Garcia JB, da Silva AA, Mualem Araújo RL, Jansen RC. Prevalence, characteristics, and factors associated with chronic pain with and without neuropathic characteristics in São Luís, Brazil. J Pain Symptom Manage. 2012;44(2):239-251.

13. Spallone V, Morganti R, D'Amato C, Greco C, Cacciotti L, Marfia GA. Validation of DN4 as a screening tool for neuropathic pain in painful diabetic polyneuropathy. Diabet Med. 2012;29(5):578-585.

14. Rabin R, de Charro F. EQ-5D: a measure of health status from the EuroQol Group. Ann Med. 2001;33(5):337-343.

15. Viegas Andrade M, Noronha K, Kind P, et al. Societal preferences for EQ-5D health states from a Brazilian population survey. Value Health Reg Issues. 2013;2(3):405-412.

16. Cleeland CS, Ryan KM. Pain assessment: global use of the Brief Pain Inventory. Ann Acad Med Singapore. 1994;23(2):129-138.

17. Reilly MC, Zbrozek AS, Dukes EM. The validity and reproducibility of a work productivity and activity impairment instrument. Pharmacoeconomics. 1993;4(5):353-365.

18. Santos JG, Brito JO, de Andrade DC, et al. Translation to Portuguese and validation of the Douleur Neuropathique 4 questionnaire. J Pain. 2010;11(5):484-490.

19. Goren A, Gross HJ, Fujii RK, Pandey A, Mould-Quevedo J. Prevalence of pain awareness, treatment, and associated health outcomes across different conditions in Brazil. Revista Dor. 2012;13(4):308-319.

20. Smith BH, Torrance N. Epidemiology of neuropathic pain and its impact on quality of life. Curr Pain Headache Rep. 2012;16(3):191-198.

21. Sadosky A, Schaefer C, Mann R, et al. Burden of illness associated with painful diabetic peripheral neuropathy among adults seeking treatment in the US: results from a retrospective chart review and cross-sectional survey. Diabetes Metab Syndr Obes. 2013;6:79-92.

22. Parsons B, Schaefer C, Mann R, et al. Economic and humanistic burden of post-trauma and post-surgical neuropathic pain among adults in the United States. J Pain Res. 2013;6:459-469.

23. Sadosky A, Schaefer C, Mann R, et al. Burden of chronic low back pain with a neuropathic pain component: retrospective chart review and cross-sectional survey among adults seeking treatment in the United States. J Pain Relief. 2014;3:163.

24. Schaefer C, Mann R, Sadosky A, et al. Burden of illness associated with peripheral and central neuropathic pain among adults seeking treatment in the United States: a patient-centered evaluation. Pain Med. 2014; 15(12):2105-2119.

25. Mann R, Sadosky A, Schaefer C, et al. Burden of HIV-related neuropathic pain in the United States. J Int Assoc Provid AIDS Care. 2016;15(2):114-125.

26. Mann R, Schaefer C, Sadosky A, et al. Burden of spinal cord injuryrelated neuropathic pain in the United States: retrospective chart review and cross-sectional survey. Spinal Cord. 2013;51(7):564-570. 
27. Doth AH, Hansson PT, Jensen MP, Taylor RS. The burden of neuropathic pain: a systematic review and meta-analysis of health utilities. Pain. 2010;149(2):338-344.

28. Rey-Ares L, Kind P, Fernandez G, et al. Self-reported population health based on EuroQol EQ-5D: population norms from Argentina, Brazil, Chile and Uruguay. Value Health. 2016;19(3):A282-A283.
29. Udall M, Kudel I, Cappelleri JC, et al. Epidemiology of physiciandiagnosed neuropathic pain in Brazil [poster abstract]; 2017. Available at: https://tools.ispor.org/ScientificPresentationsDatabase/ Presentation/74500?pdfid=50920. Accessed December 4, 2018.

\section{Publish your work in this journal}

The Journal of Pain Research is an international, peer reviewed, open access, online journal that welcomes laboratory and clinical findings in the fields of pain research and the prevention and management of pain. Original research, reviews, symposium reports, hypothesis formation and commentaries are all considered for publication.

\section{Dovepress}

The manuscript management system is completely online and includes a very quick and fair peer-review system, which is all easy to use. Visit http://www.dovepress.com/testimonials.php to read real quotes from published authors. 